\title{
Tunable ring-coupled Mach-Zehnder interferometer based on lithium niobate
}

\section{Mehmet E. Solmaz}

To cite this article: Mehmet E. Solmaz (2014) Tunable ring-coupled Mach-Zehnder interferometer based on lithium niobate, Journal of Modern Optics, 61:5, 419-423, DOI: 10.1080/09500340.2014.890254

To link to this article: https://doi.org/10.1080/09500340.2014.890254

册 Published online: 20 Feb 2014.

6 Submit your article to this journal

Щ Article views: 111

View Crossmark data $[\pi$ 


\title{
Tunable ring-coupled Mach-Zehnder interferometer based on lithium niobate
}

\author{
Mehmet E. Solmaz* \\ Institute of Materials Science and Nanotechnology, National Nanotechnology Research Center (UNAM), \\ Bilkent University, Ankara, 06800, Turkey
}

(Received 21 October 2013; accepted 29 January 2014)

\begin{abstract}
The fabrication and characterization are reported of a Fano resonance-based electro-optically tunable ring resonator-coupled Mach-Zehnder interferometer device based on a chalcogenide-lithium niobate hybrid waveguide system. The experiments reveal inherent asymmetric lineshapes that can be flipped to a near symmetrical resonance by applying a dc voltage of $\sim 10 \mathrm{~V}$ across the electrode at the MZI non-resonator arm. Further increase in voltage yields the reverse of initial asymmetry. The optical filter-based transfer-matrix model easily explains the experimental data and indicates ways to further enhance the usability of such architecture.
\end{abstract}

Keywords: lithium niobate; chalcogenide; Mach-Zehnder interferometer; tunable optical filter

\section{Introduction}

Optical waveguide systems consisting of ring resonators coupled to Mach-Zehnder interferometers (MZIs) offer the advantages of both feedforward and feedback filters. Ring resonators in an allpass filter configuration are placed on either arm of the MZI to realize flattened bandpass filters [1], add-drop filters [2], and sensing architectures [3]. In this configuration, asymmetric (Fano) resonance lineshapes occur, either due to imbalance of the MZI arms [4] or due to power coupling region design and fabrication $[5,6]$. We previously demonstrated that the pole/zero phase difference of the transfer function of an allpass ring resonator causes asymmetric resonance [7]. The physical origin of phase difference was a result of different optical losses on ring and bus waveguides, which were fabricated from different materials. The chalcogenide ring waveguide was arsenic trisulfide (AS), while the bus waveguide was lithium niobate $(\mathrm{LN})$. Here, we use the same ring resonator structure coupled to a MZI to experimentally show asymmetric lineshape tuning in a hybrid chalcogenide-lithium niobate system by electro-optical means. We further carried out simulations to model the ring-coupled MZI filter behavior. Our proposed device has potential applications in chemical sensing and optical filtering due to the ability to tailor higher sensitivity with sharp intensity response and output interchangeability.

\section{Design and fabrication of AS-on-LN hybrid waveguides}

The AS-on-LN hybrid waveguide fabrication process was described elsewhere [8]. Briefly, a titanium thickness of $110 \pm 5 \mathrm{~nm}$ was sputtered on an already diced $\mathrm{x}$-cut $y$-propagating LN chip of $5 \mathrm{~cm}$ length and $1.7 \mathrm{~cm}$ width; $7 \mu \mathrm{m}$ wide Ti strips were delineated after contact lithography on the LN chip followed by reactive-ion etching (RIE) under $\mathrm{CHF}_{3}$ and argon plasma. The two-input, two-output $(2 \times 2)$ MZI was designed to have back-toback Y-branches for the first and second coupler regions. The MZI arms were $100 \mu \mathrm{m}$ apart and the angle at the Y-branch was less than $1^{\circ}$. Ti strips were diffused for $10.25 \mathrm{~h}$ in wet breathing air ambient. The end facets of the Ti-diffused waveguide chip were then polished to optical quality. A thin film of AS was deposited on the Ti-diffused LN using RF magnetron sputtering with a $1 \AA / \mathrm{s}$ deposition rate to result in $465 \pm 14 \mathrm{~nm}$ total AS thickness. A double layer of $\mathrm{SiO}_{2}(200 \pm 10 \mathrm{~nm})$ and $\mathrm{Ti}$ $(15 \pm 3 \mathrm{~nm})$ was covered on the chalcogenide thin film using e-beam evaporation to protect the AS from chemical attack by the photoresist developer. Contact photolithography was again used to pattern on the $\mathrm{Ti}$ and a subsequent RIE step formed the AS ring structure on the Ti-diffused waveguide chip. The protective layer $\left(\mathrm{SiO}_{2}\right.$ and $\left.\mathrm{Ti}\right)$ was removed using hydrofluoric acid. The coupling region of the racetrack ring resonator was aligned right next to the Ti-diffused strip. The design of the back-to-back $1.25 \mathrm{~mm}$ long tapers followed a previous design [9] and the width went from $1 \mu \mathrm{m}$ to $3 \mu \mathrm{m}$. The radius of the $180^{\circ}$ bends was $350 \mu \mathrm{m}$ and the circumference of the racetrack structure was $7.2 \mathrm{~mm}$ to correspond to $\sim 17 \mathrm{GHz}$ free-spectral range.

The $2 \mathrm{~cm}$ long electrodes at the bottom MZI arm were patterned on both sides of the Ti-diffused waveguides. The AS-on-LN waveguide was covered with negatively patterned photoresist and a lift-off procedure

\footnotetext{
*Email: solmaz@unam.bilkent.edu.tr 
was used following e-beam evaporation of $500 \pm 20 \mathrm{~nm}$ aluminum. The electrode width and length were $13 \mu \mathrm{m}$ and $20 \mathrm{~mm}$, respectively, while the gap between the electrodes was $13 \mu \mathrm{m}$.

\section{Tunable ring-coupled MZI theory}

The AS ring-coupled MZI structure is shown in Figure 1. The racetrack ring resonator is in the allpass filter architecture and aligned on the upper arm of the MZI. In this configuration, if the racetrack feedback path is lossless, the magnitude response yields no dips. However, in practice, the loss encountered at each roundtrip of propagation breaks the pole/zero symmetry of the ideal allpass filter and creates both a wavelength-dependent amplitude and phase response. The electrode at the bottom arm creates a phase difference between MZI arms for optical signal modulation.

We can write the transfer matrix of such a ring-coupled MZI system analogous to digital filters [10] as follows,

$$
\left[\begin{array}{l}
Y_{1} \\
Y_{2}
\end{array}\right]=\left[\begin{array}{ll}
H_{11} & H_{12} \\
H_{21} & H_{22}
\end{array}\right]\left[\begin{array}{l}
X_{1} \\
X_{2}
\end{array}\right]
$$

where

$$
\begin{aligned}
& H_{11}=K c^{2}-s^{2} e^{-j \phi_{1}}, \\
& H_{12}=-j\left(K s c+s c e^{-j \phi_{1}}\right), \\
& H_{21}=-j\left(K s c+s c e^{-j \phi_{1}}\right), \\
& H_{22}=-K s^{2}+c^{2} e^{-j \phi_{1}},
\end{aligned}
$$

and $c=\sqrt{1-\kappa}$ and $s=\sqrt{1-c^{2}}$ are related to power coupling ratio $(\kappa)$ of the first and second identical MZI couplers. $\phi_{1}$ is the phase term introduced by the tuning electrodes at the bottom MZI arm. $K$ is the transfer function of the ring on the upper arm defined by

$$
K=\frac{c_{R} e^{j \phi_{2}}-\gamma_{R} z^{-1}}{1-c_{R} \gamma_{R} e^{j \phi_{2} z^{-1}}}
$$

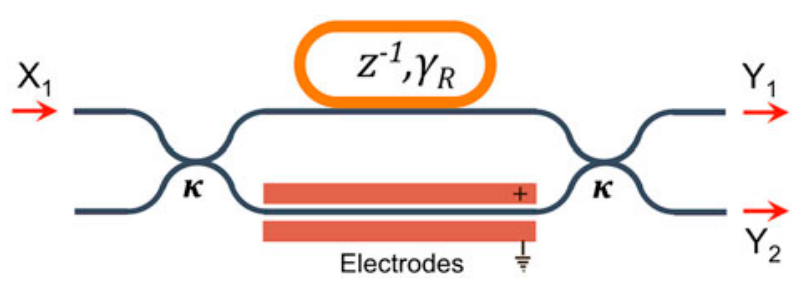

Figure 1. Schematic diagram of the ring-coupled MZI. The MZI has two equal-length arms with upper waveguide coupled to an AS race-track ring resonator and lower waveguide integrated with electrodes, enabling electro-optical phase shift. (The colour version of this figure is included in the online version of the journal.) where $z=e^{-j \beta L}$, in which $\beta$ is the propagation constant and $L$ is the ring circumference, and $\gamma_{R}=10^{-\alpha L / 20}$, where $\alpha L$ is the ring roundtrip loss. $\kappa_{R}$ is the ratio of power coupled to ring and $c_{R}=\sqrt{1-\kappa_{R}} . \phi_{2}$ is the phase difference between the pole and zero of transfer function. Although $\phi_{2}$ is zero in an ideal resonator system, it is considered significant due to the unequal waveguide coupler architecture of the AS-on-LN hybrid waveguide system [7].

If we have $X_{1}=1$ and $X_{2}=0$, input-output transfer functions can be written as follows:

$$
\frac{Y_{1}}{X_{1}}=\frac{\left(c^{2} c_{R} e^{j \phi_{2}}-s^{2} e^{-j \phi_{1}}\right)-\left(c^{2} \gamma_{R}-s^{2} c_{R} \gamma_{R} e^{-j\left(\phi_{1}-\phi_{2}\right)}\right) z^{-1}}{1-c_{R} \gamma_{R} e^{j \phi_{2} z^{-1}}}
$$

$\frac{Y_{2}}{X_{1}}=-j \frac{\left(s c c_{R} e^{j \phi_{2}}+s c e^{-j \phi_{1}}\right)-\left(s c \gamma_{R}+s c c_{R} \gamma_{R} e^{-j\left(\phi_{1}-\phi_{2}\right)}\right) z^{-1}}{1-c_{R} \gamma_{R} e^{j \phi_{2} z^{-1}}}$.

\section{Electro-optic characterization of AS-on-LN hybrid waveguides}

Optical testing of the AS-on-LN hybrid waveguide platform was performed using fiber end-coupling from both LN facets on an alignment stage. The magnitude and group delay responses were measured using a LUNA Technologies optical vector analyzer (OVA), which carries a tunable laser source and coherent detector inside. The OVA measured the full Jones matrix of the waveguide chip under test and the results of both TE and TM polarizations were obtained.

We measured the Ti-diffused LN MZI structure without AS ring and bottom electrode to obtain the insertion loss. $X_{1}-\left(Y_{1}+Y_{2}\right)$ and $X_{2}-\left(Y_{1}+Y_{2}\right)$ losses were measured to be $\sim 6 \mathrm{~dB}$, while the straight Ti-diffused LN waveguide loss next to the MZI was $\sim 2.5 \mathrm{~dB}$. The excess loss is contributed to by two coupler regions. After full integration of AS ring resonator and aluminum electrode, the electrode probes were fed by a tunable dc power supply to observe electro-optical behavior. As a result of Pockel's effect in LN, the phase change at the bottom MZI arm is a function of applied voltage, electrode gap, electric/optical field overlap, and electrode length. The electro-optical coefficient of interest for TM polarization in x-cut $y$-propagating $\mathrm{LN}$ is $r_{13}=9.6 \mathrm{pm} / \mathrm{V}$. The dc voltage was increased in $5 \mathrm{~V}$ increments, and the magnitude and group delay responses with single input $\left(X_{1}\right)$ at the bar $\left(Y_{1}\right)$ and cross $\left(Y_{2}\right)$ ports were recorded.

Figure $2(a)$ shows the magnitude response of TM polarization at bar (in red) and cross (in blue) ports as a function of applied dc voltage. When there is no applied voltage, the spectrum exhibits asymmetric behavior, which is a result of both potential imbalance on MZI 

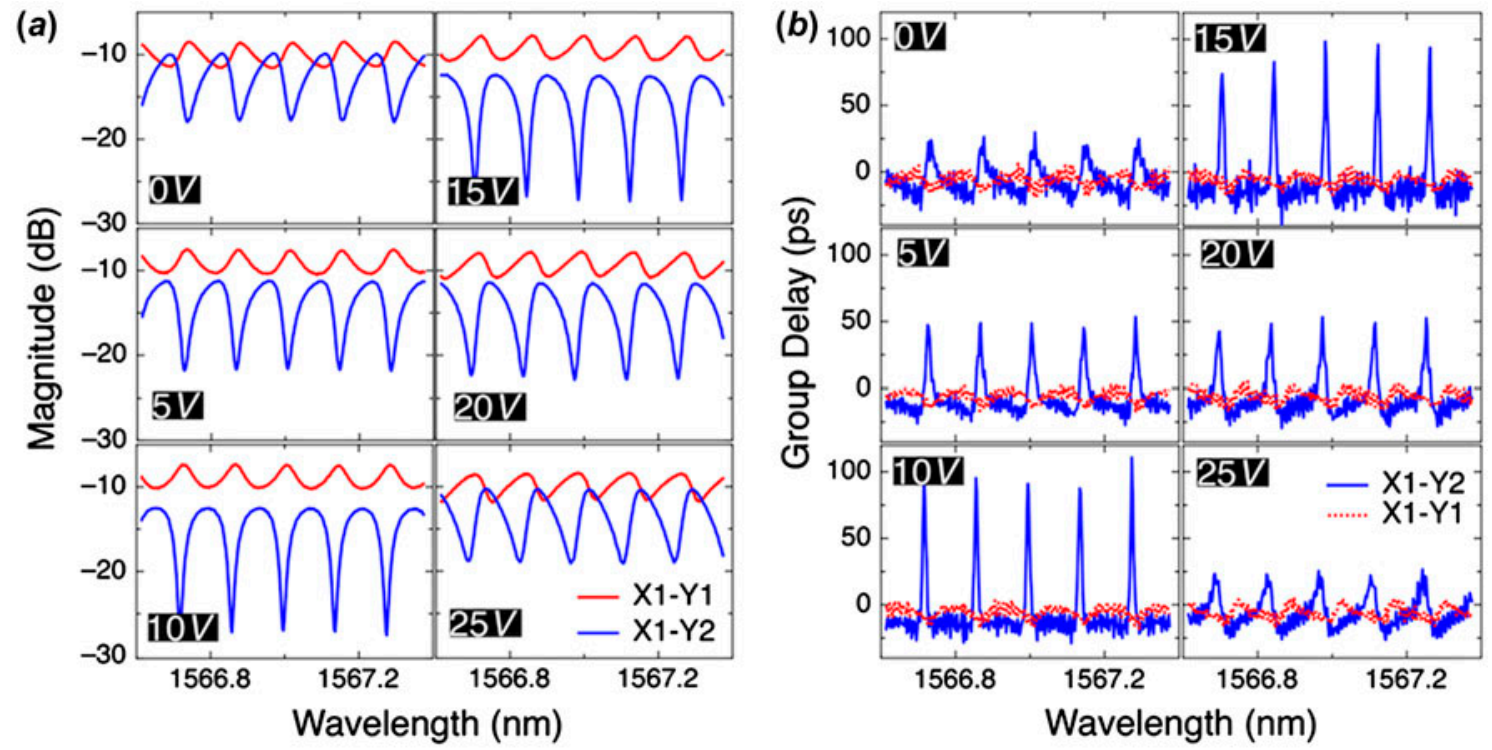

Figure 2. Transmission magnitude $(a)$ and group delay response (b) through bar (red) and cross (blue) ports as a function of applied voltage on the lower arm of the MZI. The voltage was increased from $0 \mathrm{~V}$ to $25 \mathrm{~V}$ in $5 \mathrm{~V}$ increments. (The colour version of this figure is included in the online version of the journal.)

arms and the phase shift between ring resonator pole and zero. The imbalance on MZI arms may stem from unequal MZI arm lengths that could be a result of imperfect mask design or fabrication imperfections [4], and is inherent in $\phi_{1}$. To measure the phase imbalance, the MZI portion of the filter was tested before the fabrication of the AS ring structure and aluminum electrodes, and the optical spectrum showed no filtering behavior on both bar and cross ports. Therefore we eliminated the possibility of unequal lengths for MZI arms and assumed $\phi_{1}=0$ at $V=0$.

The pole/zero phase difference is expressed by $\phi_{2}$. Although the bar port extinction ratio stays the same $(\sim 3 \mathrm{~dB})$ for all applied voltages, the cross port extinction ratio goes from $\sim 8 \mathrm{~dB}$ to $\sim 14.6 \mathrm{~dB}$ as the voltage goes from $0 \mathrm{~V}$ to $10 \mathrm{~V}$, and returns to $\sim 8 \mathrm{~dB}$ at $25 \mathrm{~V}$. The asymmetric lineshape at both ports diminishes and flips to symmetrical around $10 \mathrm{~V}$ and reverses itself as the voltage increases to $25 \mathrm{~V}$ though the asymmetry is more obvious at the cross port than at the bar port. With a measurement resolution of $2.5 \mathrm{pm}$, the cross port resonances experience a wavelength shift of $10.0 \pm 2.5 \mathrm{pm}$ for each $5 \mathrm{~V}$ increase, while the dips at the bar port shift $15.0 \pm 2.5 \mathrm{pm}$ for each $5 \mathrm{~V}$ increase.

Figure 2(b) shows the measured group delay for bar and cross ports. The group delay becomes larger with the extinction ratio and loses its asymmetric behavior with further increase in voltage. Since the group delay near the resonance is positive, the zero magnitude of $Y_{2} / X_{1},\left|z_{12}\right|$, is bigger than one and outside the unit circle. A positive group delay of $Y_{2} / X_{1}$ means that the optical filter is a maximum-phase filter. Yet, from Equation (5) it is clear that the zero magnitude cannot be bigger than one due to every $z$-term being multiplied by $\gamma_{R}$, which is also less than 1 . Positive group delay can be justified by adding loss terms on upper $\left(\gamma_{1}\right)$ and lower $\left(\gamma_{2}\right)$ MZI arms in the first and second terms in Equation (2), respectively. Optical losses are the outcome of fabrication processes and can be improved by adjusting waveguide design and fabrication parameters. The zero magnitude of $Y_{1} / X_{1},\left|z_{11}\right|$, is less than one due to marginally negative group delay. The optical filter is a minimum-phase filter at this port.

From Equations (4) and (5), we see that the phase of the transfer function pole $\left(\angle p=\phi_{2}\right)$ is a constant introduced by the AS ring resonator coupling to Ti-diffused LN. However, increase in voltage or $\phi_{1}$ makes the phase of the transfer function zero $\left(\angle z_{12}\right)$ change and eventually achieve $\left(\angle z_{12}-\phi_{2}\right) \rightarrow 0$. Further increase in voltage separates $\phi_{1}$ from $\phi_{2}$ even further and causes reversal of the asymmetric behavior. We simulated the asymmetric behavior of the ring-coupled MZI system using the filter model proposed. We chose $50 \%$ couplers for the MZI, 90\% coupling to the AS ring, $1 \mathrm{~dB}$ of roundtrip loss in the ring, and $\pi / 8$ phase difference between zero and pole of the AS ring. The simulation of the model is presented in Figure 3.

We assume that the increasing lower arm voltage creates a phase shift between 0 and $\pi$. We look at the magnitude and phase responses of pole and zero as a function of lower arm phase shift. Both $\angle z_{11}$ and $\angle z_{12}$ increase with $\phi_{1}$, and $\angle z_{12}$ crosses $\angle p$ at 0.71 radians 
while $\angle z_{11}+\pi$ crosses $\angle p$ at 0.23 radians (Figure $3(a)$ ). The reason $\angle z_{11}+\pi$ is plotted instead of $\angle z_{11}$ is obvious from Figure $3(b)$, which depicts the zero locations at $\phi_{1}=0, \phi_{1}=0.23$, and $\phi_{1}=0.71$. When $\phi_{1}=0.23$, $\angle z_{11}$ and $\angle p$ are on the same line passing through the origin. $Y_{1} / X_{1}$ becomes fully symmetric unlike the $Y_{2} / X_{1}$ transmission response (Figure 3(c)). When $\phi_{1}=0.71$, $Y_{2} / X_{1}$ becomes fully symmetric, and $\angle z_{12}$ and $\angle p$ are on the same line passing through the origin, while $Y_{1} / X_{1}$ again becomes asymmetric. The $\phi_{1}$ change on the lower arm also shifts the resonance wavelength, similar to experimental results.

The power coupling ratios $\left(\kappa, \kappa_{R}\right)$ can be modified by using different waveguide design and can give different filtering characteristics and tunability. However, the inherent AS ring resonator pole-zero phase difference will always cause asymmetric behavior for a ring-coupled MZI system and optical losses stemming from fabrication parameters only change the spectral response of the filter. For instance, adjusting the ring roundtrip $\left(\gamma_{R}\right)$ loss does not tune the asymmetric behavior, hence $\angle z_{11}$ and $\angle z_{12}$. It only changes the sharpness at filter magnitude response. Introducing the same optical loss on both MZI arms similarly has no effect on the asymmetric behavior, filter extinction ratio, and filter sharpness, but offsets the overall filter response by the same amount. Nevertheless, introducing unequal loss on MZI arms modifies the amount of phase shift necessary on the lower MZI arm to correct the asymmetric behavior on both ports. Consequently, the ring-coupled MZI filter response can be tuned with phase change on the lower MZI arm, leading to distinctive phase changes for pole and zero of the filter transfer function, unlike in an allpass ring resonator structure, where the zero and pole magnitudes are unchanged while their phases can be modified the same amount if a phase shifter is placed on the resonator feedback path.

Ti-diffused LN waveguides have advantage over small cross-sectional area waveguide systems (silicon, silica, III-V compounds) in terms of coupling to single-mode fiber operation at telecommunications wavelengths. The typical coupling loss is less than $1 \mathrm{~dB}$, whereas the coupling loss of silicon core waveguides is $\sim 3.5 \mathrm{~dB}$ with tapered mode size converter [11]. Another advantage is the electro-optical effect that gives tuning capability for feedforward optical filters. However, Ti-diffused LN waveguides lack the ability to have highly bent structures due to low core-to-cladding index contrast. The AS ring structure brings feedback filter structure to an already established waveguide material. As a future application, the device presented can be used as a high-speed add-drop filter with switchable outputs if the coupling regions are optimized to be $3 \mathrm{~dB}$ couplers [2], and AS ring waveguide loss is minimized. Tunability
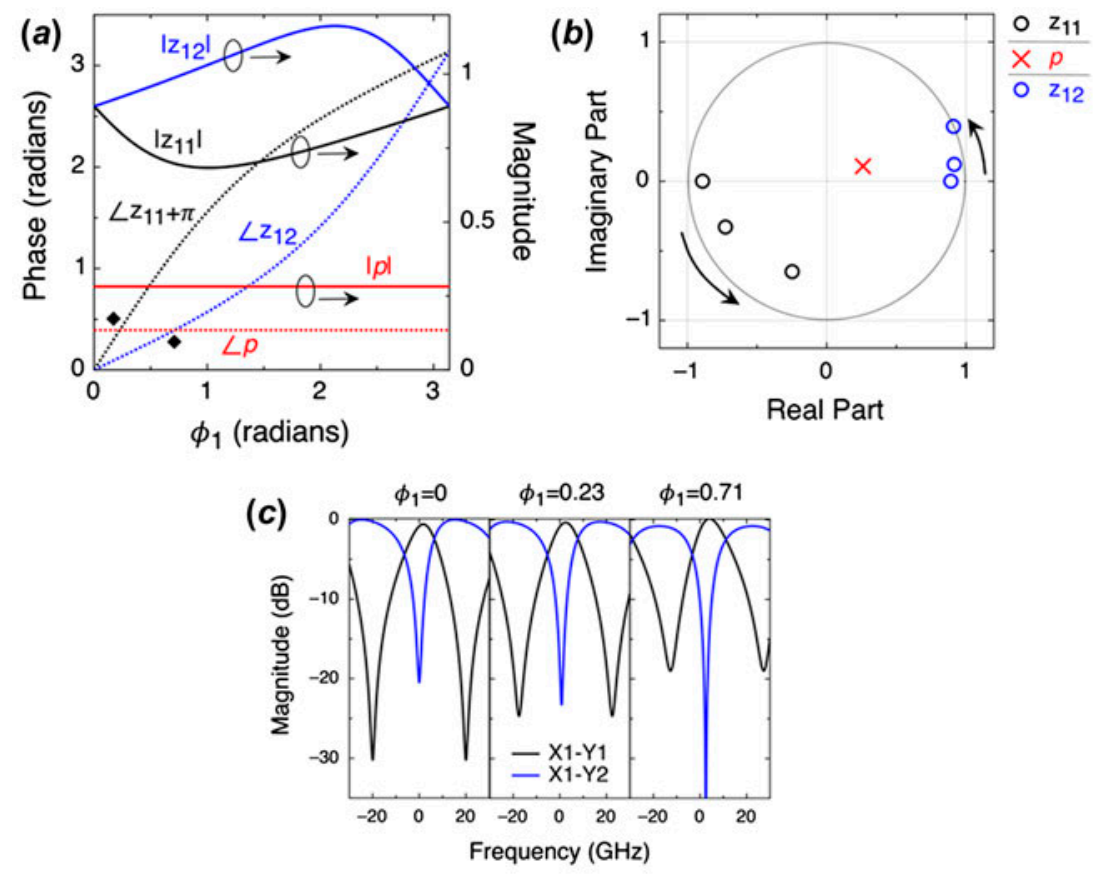

Figure 3. Simulation of tunable ring-coupled MZI waveguide structure as a function of phase shift induced in the lower MZI arm $\left(\phi_{1}\right)$. (a) Pole-zero magnitude and phase as a function of $\phi_{1}$. (b) Pole-zero locations on unit circle as $\angle z_{12}$ and $\angle z_{11}+\pi$ intersect with $\angle p$. (c) Magnitude response at $\phi_{1}=0, \phi_{1}=0.23$, and $\phi_{1}=0.71$ radians. (The colour version of this figure is included in the online version of the journal.) 
of asymmetric behavior may also be useful in biosensor applications where the wavelength is biased on a sharp slope and the output power is the sensitivity marker. The possible drift on the slope can be corrected by adjusting phase on the lower arm.

In conclusion, we have reported the fabrication and characterization of an electro-optically tunable ringcoupled MZI device based on a chalcogenide-lithium niobate hybrid waveguide system that is capable of generating Fano-shaped resonances. The analytical model of the transfer function of the ring-coupled MZI was used to explain the asymmetry and tunability. The inherent phase difference between the pole and zero of the AS ring resonator introduces an asymmetric lineshape, which can be tuned using the electrode on the opposing MZI arm. Introducing phase change using the electro-optical effect modifies the magnitude and phase of the transfer function zeros, and asymmetry, extinction ratio, and sharpness of the filter were altered. We elaborated on the effects of design and fabrication parameters on the filter response. A modified design and improved fabrication processes will make this intriguing device suitable for add-drop filters in wavelength division multiplexing systems and sensing systems based on intensity variation.

\section{Acknowledgement}

The author acknowledges Dr. Christi K. Madsen and the Photonic Signal Processing research group at Texas A\&M
University for fruitful discussions, and help in characterization of the device.

\section{Funding}

This work was supported by the Scientific and Technological Research Council of Turkey (TUBITAK) [grant number BIDEB-2232].

\section{References}

[1] Madsen, C.K. IEEE Photonics Technol. Lett. 1998, 10, $1136-1138$.

[2] Zhou, L.; Poon, A.W. Opt. Lett. 2007, 32, 781-783.

[3] Terrel, M.; Digonnet, M.J.; Fan, S. Appl. Opt. 2009, 48, 4874-4879.

[4] Absil, P.; Hryniewicz, J.; Little, B.; Wilson, R.; Joneckis, L.; Ho, P.-T. IEEE Photonics Technol. Lett. 2000, 12, 398-400.

[5] Ruege, A.C.; Reano, R.M. J. Lightwave Technol. 2009, 27, 2035-2043.

[6] Darmawan, S.; Tobing, L.Y.M.; Mei, T. Opt. Lett. 2010, $35,238-240$.

[7] Solmaz, M.E.; Zhou, Y.; Madsen, C.K. J. Lightwave Technol. 2010, 28, 2951-2955.

[8] Solmaz, M.E.; Adams, D.B.; Grover, S.; Tan, W.C.; Xia, X.; Eknoyan, O.; Madsen, C.K. IEEE Photonics Technol. Lett. 2009, 21, 557-559.

[9] Solmaz, M.E.; Adams, D.B.; Tan, W.C.; Snider, W.T.; Madsen, C.K. Opt. Lett. 2009, 34, 1735-1737.

[10] Madsen, C.K.; Zhao, J.H. Optical Filter Design and Analysis: a Signal Processing Approach; John Wiley \& Sons: New York, 1999.

[11] Shoji, T.; Tsuchizawa, T.; Watanabe, T.; Yamada, K.; Morita, H. Electron. Lett. 2002, 38, 1669-1670. 